\title{
Accelerating Convergence of Eigenfunction Expansions
}

\author{
By J. K. Shaw, L. W. Johnson and R. D. Riess
}

\begin{abstract}
A general procedure is presented for accelerating the convergence of eigenfunction expansions associated with selfadjoint boundary-value problems. The results obtained reduce, in special cases, to certain well-known methods of acceleration, including the Lanczos representation. The generality of the procedure allows the user to take advantage of structural properties of the expanded function.
\end{abstract}

1. Introduction. In this paper we shall be concerned with orthogonal expansions

$$
f=\sum_{k=1}^{\infty} a_{k} y_{k},
$$

where the $y_{k}$ are eigenfunctions of a selfadjoint boundary-value problem on an interval $[a, b]$, and where the $a_{k}$ are corresponding generalized Fourier coefficients of $f$. It is well known [3] that such expansions always converge at least in the norm of $L^{2}[a, b]$. However, depending on smoothness and boundary behavior of $f,(1.1)$ may or may not actually represent $f(x)$ pointwise in $[a, b]$. Moreover, the rate of convergence of (1.1) may be so slow that the representation is of little use for purposes of approximating $f$.

The purpose of this paper is to develop a modified eigenfunction expansion which represents $f(x)$ pointwise in $[a, b]$, and whose coefficients tend to 0 with arbitrary rapidity. Given a suitably smooth function $f$ and a positive integer $p$, we introduce a function $h_{p}$, depending only on $f$ and $p$, such that

$$
f(x)=h_{p}(x)+\sum_{k=1}^{\infty} \hat{a}_{k} y_{k}(x), \quad a \leqslant x \leqslant b,
$$

with uniform convergence in $[a, b]$, and such that the "modified" coefficients $\hat{a}_{k}$ have order of magnitude $\lambda_{k}^{-p}$, where $\lambda_{k}$ is the $k$ th eigenvalue of the boundary-value problem. Since $\left|\lambda_{k}\right| \rightarrow \infty$, as $k \rightarrow \infty$, we say that the rate of convergence of (1.1) has been "accelerated" by means of (1.2).

In the context of ordinary Fourier series, the notion of accelerated convergence has been studied in some detail. The most familiar result is the Lanczos representation of functions in terms of Bernoulli polynomials and trigonometric series (Lanczos [5], Lyness [6], Jones and Hardy [4]). This representation for functions $f \in C^{p}[0,1]$, $p \geqslant 1$, has the form

Received February 11, 1975; revised September 29, 1975.

AMS (MOS) subject classifications (1970). Primary 42A16, 41 A10; Secondary 65D15.

Key words and phrases. Eigenfunction expansions, acceleration of convergence, Lanczos representation, selfadjoint linear differential operators. 


$$
\begin{aligned}
f(x)= & \sum_{k=0}^{p-1}\left[f^{(k)}(1)-f^{(k)}(0)\right] \frac{B_{k+1}(x)}{k !}+\int_{0}^{1} f(t) d t \\
& +2 \sum_{k=1}^{\infty}\left[c_{k} \cos 2 k \pi x+s_{k} \sin 2 k \pi x\right],
\end{aligned}
$$

where $B_{k}$ is the $k$ th Bernoulli polynomial and where

$$
c_{k}+i s_{k}=\frac{(-1)^{p}}{(2 k \pi i)^{p}} \int_{0}^{1} f^{(p)}(t)\left(e^{2 k \pi i t}-1\right) d t, \quad k=1,2,3, \ldots
$$

A similar type of representation, which involves only sine terms, has been used by Jones and Hardy [4] as an approximation method. This expansion may be written

$$
f(x)=\sum_{k=0}^{p-1}\left[f^{(2 k)}(1) A_{k}(x)+f^{(2 k)}(0) A_{k}(1-x)\right]+\sum_{k=1}^{\infty} \alpha_{k} \sin k \pi x,
$$

where

$$
\alpha_{k}=\frac{(-1)^{p}}{(k \pi)^{2 p}} \int_{0}^{1} 2 f^{(2 p)}(t) \sin k \pi t d t
$$

and where $A_{k}$ is the Lidstone polynomial [2] of degree $2 k+1$. The derivations of (1.3) and (1.4) require no more than integration by parts and using properties of the polynomials involved.

The representation (1.2), which we now proceed to derive, includes (1.3), (1.4) and various other representations as special cases. However, the overall approach we take still recalls Lanczos' original idea of modification of boundary behavior of the expanded function. The essential point of departure is that we are able to pass from the method of integration by parts to the more general setting of selfadjointness in linear differential operators.

2. The Generalized Representation. Let $n$ be a fixed positive integer and let $L$ be the $n$th order linear differential operator given by

$$
L y=a_{0} y^{(n)}+a_{1} y^{(n-1)}+\cdots+a_{n-1} y^{\prime}+a_{n} y,
$$

where each $a_{j}$ is a complex-valued function of class $C^{n-j}$ on the closed interval $a \leqslant x$ $\leqslant b$, and $a_{0}(t) \neq 0$ for $t \in[a, b]$. Suppose we are given linearly independent boundary forms

$$
B_{j} y=\sum_{k=1}^{n} M_{j k} y^{(k-1)}(a)+N_{j k} y^{(k-1)}(b), \quad 1 \leqslant j \leqslant n,
$$

where the $M_{j k}$ and $N_{j k}$ are complex constants, and let us agree that $B y=0$ shall denote the set of conditions $B_{1} y=B_{2} y=\cdots=B_{n} y=0$. We shall suppose throughout that the eigenvalue problem

$$
L y=\lambda y, \quad B y=0
$$

is selfadjoint; that is, $(L u, v)=(u, L v)$ whenever $B u=B v=0$, where $(\cdot, \cdot)$ is the usual inner product $(f, g)=\int_{a}^{b} f(t) \overline{g(t)} d t$. Then the eigenvalues $\left\{\lambda_{k}\right\}_{1}^{\infty}$ of $(2.1)$ comprise a real, countably infinite set with no finite limit point. Denoting by $\left\{y_{k}\right\}_{1}^{\infty}$ the corresponding set of normalized eigenfunctions,

$$
L y_{k}=\lambda_{k} y_{k}, \quad B y_{k}=0, \quad\left\|y_{k}\right\|_{2}=1,
$$


the eigenfunction expansion (1.1) may be written

$$
f=\sum_{k=1}^{\infty}\left(f, y_{k}\right) y_{k} .
$$

To derive the modified version of (2.2), we must consider separately the two cases arising from whether or not 0 is an eigenvalue of (2.1). Mainly, the presentation we give is for the case in which 0 is not an eigenvalue. To avoid undue repetition, we shall simply state the pertinent results for the other case and leave the details of proof to the reader.

Thus suppose first that 0 is not an eigenvalue of (2.1). Then for any fundamental system of solutions $\varphi_{1}, \varphi_{2}, \ldots, \varphi_{n}$ of the homogeneous equation $L y=0$, the rank of the $(n \times n)$ matrix $\left(B_{j} \varphi_{k}\right)$ is $n$. Therefore, there exist unique functions $p_{1}, p_{2}, \ldots, p_{n}$ such that

$$
L p_{k}=0, \quad B_{j} p_{k}=\delta_{j k}, \quad 1 \leqslant j, k \leqslant n .
$$

Now define the functions $p_{n+1}, p_{n+2}, \ldots$ by the recurrence formula

$$
L p_{n k+j}=p_{n(k-1)+j}, \quad B p_{n k+j}=0
$$

for $1 \leqslant j \leqslant n$ and $k=1,2,3, \ldots$

LEMMA 1. Let $p$ be a positive integer. Then the functions

$$
\left\{p_{n k+j}\right\}, \quad 1 \leqslant j \leqslant n, 0 \leqslant k \leqslant p-1
$$

are linearly independent solutions of $L^{p} y=0$. Moreover, the unique solution to the problem

$$
L^{p} y=0, \quad B_{j} L^{k} y=A_{n k+j}, \quad 1 \leqslant j \leqslant n, 0 \leqslant k \leqslant p-1,
$$

is given by

$$
y=\sum_{k=0}^{p-1} \sum_{j=1}^{n} A_{n k+j} p_{n k+j}
$$

Proof. It is clear from (2.3) and (2.4) that $L^{p} p_{n k+j}=0$ for $k=0,1,2$, $\ldots, p-1$. To prove linear independence let $\left\{h_{n k+j}\right\}, 1 \leqslant j \leqslant n, 0 \leqslant k \leqslant$ $p-1$, be a sequence of constants such that

$$
\sum_{k=0}^{p-1} \sum_{k=1}^{n} h_{n k+j} p_{n k+j}=0 \text {. }
$$

If we apply the functional $B_{m} L^{r}$ to this equation, where $1 \leqslant m \leqslant n$ and $0 \leqslant r$ $\leqslant p-1$, and use (2.3) and (2.4), there follows

$$
0=B_{m} L^{r}(0)=B_{m}\left(\sum_{k=r}^{p-1} \sum_{j=1}^{n} h_{n k+j} p_{n(k-r)+j}\right)=h_{n r+m} .
$$

Hence the solutions are linearly independent. Consequently, every solution to $L^{p} y=0$ may be expressed as

$$
y=\sum_{k=0}^{p-1} \sum_{j=1}^{n} a_{n k+j} p_{n k+j}
$$

for certain constants $\left\{a_{k}\right\}_{1}^{p n}$. Arguing as in (2.6), we apply the functional $B_{m} L^{r}$ 
to the above equation and invoke condition (2.5). This results in $A_{n r+m}=$ $B_{m} L^{r} y=a_{n r+m}$, and the proof is complete.

Definition. For each nonnegative integer $m$, let $D_{m}$ be the set of all complex functions $f$ defined on $[a, b]$ such that $\left(L^{k} f\right)(x)$ and $B_{j} L^{k} f$ all exist for $0 \leqslant k \leqslant m$ and $1 \leqslant j \leqslant n$.

Theorem 1. Let $p$ be a positive integer and let $f \in D_{p}$. Then

$$
f(x)=h_{p}(x)+g_{p}(x), \quad a \leqslant x \leqslant b,
$$

where

and

$$
h_{p}(x)=\sum_{k=0}^{p-1} \sum_{j=1}^{n}\left(B_{j} L^{k} f\right) p_{n k+j}(x)
$$

$$
g_{p}(x)=\sum_{k=1}^{\infty} \frac{\left(L^{p} f, y_{k}\right)}{\lambda_{k}^{p}} y_{k}(x)
$$

Proof. Let $h_{p}$ be the unique solution to the problem

$$
L^{p} y=0, \quad B_{j} L^{k} y=B_{j} L^{k} f, \quad 1 \leqslant j \leqslant n, 0 \leqslant k \leqslant p-1 .
$$

The existence of $h_{p}$ is assured by Lemma 1 . Let $g_{p}(x)=f(x)-h_{p}(x)$, so that

$$
B_{j} L^{k} g_{p}=0, \quad 1 \leqslant j \leqslant n, 0 \leqslant k \leqslant p-1 .
$$

Since $g_{p}$ satisfies the homogeneous boundary conditions, it admits the eigenfunction expansion [3]

$$
g_{p}(x)=f(x)-h_{p}(x)=\sum_{k=1}^{\infty}\left(f-h_{p}, y_{k}\right) y_{k}(x), \quad a \leqslant x \leqslant b,
$$

with uniform convergence in $[a, b]$. By selfadjointness, the coefficients may also be written

$$
\begin{aligned}
\left(f-h_{p}, y_{k}\right) & =\lambda_{k}^{-1}\left(f-h_{p}, L y_{k}\right) \\
& =\lambda_{k}^{-1}\left(L\left(f-h_{p}\right), y_{k}\right)=\lambda_{k}^{-2}\left(L^{2}\left(f-h_{p}\right), y_{k}\right) \\
& : \\
& \cdot \\
& =\lambda_{k}^{-p}\left(L^{p}\left(f-h_{p}\right), y_{k}\right)=\lambda_{k}^{-p}\left(L^{p} f, y_{k}\right) .
\end{aligned}
$$

Thus (2.8) is equivalent to

$$
f(x)=h_{p}(x)+\sum_{k=1}^{\infty} \lambda_{k}^{-p}\left(L^{p} f, y_{k}\right) y_{k}(x)
$$

Finally, we know from Lemma 1 that

and this completes the proof.

$$
h_{p}(x)=\sum_{k=0}^{p-1} \sum_{j=1}^{n}\left(B_{j} L^{k} f\right) p_{n k+j}(x)
$$

The following formula is sometimes useful in calculating the Fourier coefficients in (2.9).

Lemma 2. Let $f \in D_{p}$. Then

$$
\lambda_{r}^{-p}\left(L^{p} f, y_{r}\right)=\left(f, y_{r}\right)-\sum_{k=0}^{p-1} \sum_{j=1}^{n} \lambda_{r}^{-k} B_{j} L^{k} f\left(p_{j}, y_{r}\right)
$$


Proof. Let $r$ be fixed. If we take the inner product of the series

$$
f(x)=\sum_{k=0}^{p-1} \sum_{j=1}^{n}\left(B_{j} L^{k} f\right) p_{n k+j}(x)+\sum_{k=1}^{\infty} \lambda_{k}^{-p}\left(L^{p} f, y_{k}\right) y_{k}(x)
$$

with the eigenfunction $y_{r}$, we obtain

$$
\left(f, y_{r}\right)=\sum_{k=0}^{p-1} \sum_{j=1}^{n}\left(B_{j} L^{k} f\right)\left(p_{n k+j}, y_{r}\right)+\lambda_{r}^{-p}\left(L^{p} f, y_{r}\right) .
$$

In view of (2.4), we have

$$
\begin{aligned}
\left(p_{n k+j}, y_{r}\right) & =\lambda_{r}^{-1}\left(p_{n k+j}, L y_{r}\right) \\
& =\lambda_{r}^{-1}\left(p_{n(k-1)+j}, y_{r}\right)=\lambda_{r}^{-2}\left(p_{n(k-2)+j}, y_{r}\right) \\
& : \\
& \cdot \\
& =\lambda_{r}^{-k}\left(p_{j}, y_{r}\right) .
\end{aligned}
$$

Substituting this into (2.11) results in an equation which is equivalent to (2.10).

We conclude this section with statements of corresponding results for the case in which 0 is a simple eigenvalue of (2.1). For details concerning the theoretical basis of this method we refer the reader to a recent paper of the first author [7, Section 5].

Thus suppose that 0 is a simple eigenvalue of $(2.1)$ and let $y_{0}$ be a normalized eigenfunction; i.e.,

$$
L y_{0}=0, \quad B y_{0}=0, \quad\left\|y_{0}\right\|_{2}=1 \text {. }
$$

For each fundamental system of solutions $\varphi_{1}, \varphi_{2}, \ldots, \varphi_{n}$ of $L y=0$, the rank of the matrix $\left(B_{j} \varphi_{k}\right)_{j, k=1}^{n}$ is $n-1$. Since rank is independent of the choice of the $\varphi_{k}$, we may take $\varphi_{1}=y_{0}$. Further, we may suppose that, after possibly re-ordering the forms $B_{1}, B_{2}, \ldots, B_{n}$, the last $n-1$ rows of the matrix are linearly independent. Then, if $n>1$, there exist uniquely determined functions $q_{2}, q_{3}, \ldots, q_{n}$ such that

$$
L q_{k}=0, \quad B_{j} q_{k}=\delta_{j k}, \quad\left(q_{k}, y_{0}\right)=0
$$

for $2 \leqslant j, k \leqslant n$. Moreover, one can show (see [7]) that there exist a function $q_{1}$ and a constant $c \neq 0$ such that

$$
L q_{1}=c y_{0}, \quad B_{k} q_{1}=\delta_{k 1}, \quad\left(q_{1}, y_{0}\right)=0
$$

for $1 \leqslant k \leqslant n$. Define the auxiliary boundary forms $\left\{U_{j}\right\}_{1}^{n}$ by

$$
\begin{aligned}
& U_{1}=B_{1}-\left[\left(B_{1} q_{2}\right) B_{2}+\left(B_{1} q_{3}\right) B_{3}+\cdots+\left(B_{1} q_{n}\right) B_{n}\right], \\
& U_{k}=B_{k}, \quad 2 \leqslant k \leqslant n,
\end{aligned}
$$

and note that, by (2.12) and (2.13),

$$
U_{j} q_{k}=\delta_{j k}, \quad\left(q_{k}, y_{0}\right)=0, \quad U q_{n k+j}=0
$$

for $1 \leqslant j \leqslant n$ and $1 \leqslant k<\infty$. Then, in analogy to Theorem 1 , one may establish the following representation.

THEOREM 2. Suppose that 0 is a simple eigenvalue of (2.1), let $p$ be a positive integer, and let $f \in D_{p}$. Then

$$
f(x)=h_{p}(x)+g_{p}(x), \quad a \leqslant x \leqslant b,
$$


where

$$
h_{p}(x)=\left(f, y_{0}\right) y_{0}(x)+\sum_{k=0}^{p-1} \sum_{j=1}^{n}\left(U_{j} L^{k} f\right) q_{n k+j}(x)
$$

and

$$
g_{p}(x)=\sum_{k=1}^{\infty} \lambda_{k}^{-p}\left(L^{p} f, y_{k}\right) y_{k}(x),
$$

with uniform convergence in $[a, b]$.

3. Examples and Applications. The representation $f(x)=h_{p}(x)+g_{p}(x)$, from either Theorem 1 or Theorem 2 , resolves an arbitrary function $f \in D_{p}$ into a polynomial part (typically) and a generalized Fourier series. Since the Fourier series converges "rapidly", in the sense that its coefficients have order of magnitude $\lambda_{k}^{-p}$, then we may expect that the truncated expansion

$$
f(x) \cong h_{p}(x)+\sum_{k=1}^{m} \lambda_{k}^{-p}\left(L^{p} f, y_{k}\right) y_{k}(x)
$$

will yield a close approximation to $f(x)$. Another notable aspect of this procedure is that we are completely free to choose the operator $L$ and the boundary forms $B_{1}, B_{2}$, $\ldots, B_{n}$ which most efficiently utilize the structural properties of the given function $f$.

To illustrate our results, some specific examples will now be presented. We will show that all of the approximation methods in [4] and [6] are special cases of either Theorem 1 or Theorem 2 , and are attainable by choosing $L$ and $B_{1}, B_{2}, \ldots$ appropriately. Finally, to illustrate further the flexibility of our approach, we introduce a new approximation method designed especially for evaluating (approximately) the error function.

Examples. (1) Consider the representation (1.4). The Lidstone polynomials are defined inductively by $A_{0}(x)=x$ and $A_{k}^{\prime \prime}(x)=A_{k-1}(x), A_{k}(0)=A_{k}(1)=0$ for $k \geqslant 1$. Let $L y=-y^{\prime \prime}, B_{1} y=y(0)$ and $B_{2} y=y(1)$. For this data, the eigenvalues of (2.1) are $\lambda_{k}=k^{2} \pi^{2}, k=1,2,3, \ldots ;$ and the eigenfunctions are $y_{k}(x)=\sqrt{2} \sin k \pi x$. By (2.3) and (2.4) we find $p_{1}(x)=1-x, p_{2}(x)=x, p_{3}(x)=x^{3} / 6-x^{2} / 2+x / 3$, etc. In general, $p_{2 k+1}(x)=(-1)^{k} A_{k}(1-x)$ and $p_{2 k+2}(x)=(-1)^{k} A_{k}(x), k=0,1,2$, $\ldots$ By Theorem 1 , each $f \in D_{p}$ admits the representation

$$
\begin{aligned}
f(x)= & \sum_{k=0}^{p-1}\left\{(-1)^{k} f^{(2 k)}(0)(-1)^{k} A_{k}(1-x)+(-1)^{k} f^{(2 k)}(1)(-1)^{k} A_{k}(x)\right\} \\
& +\sum_{k=1}^{\infty}\left\{\int_{0}^{1}(-1)^{p} f^{(2 p)}(t) \sqrt{2} \sin k \pi t d t\right\} \frac{\sqrt{2} \sin k \pi x}{\left(k^{2} \pi^{2}\right)^{p}}
\end{aligned}
$$

and this is equivalent to (1.4).

(2) To obtain the Lanczos representation, let us recall that the Bernoulli polynomials may be defined by the relations $B_{0}(x)=1, B_{1}(1)-B_{1}(0)=1, B_{k}^{\prime}(x)=$ $k B_{k-1}(x)$ and $B_{k}(1)-B_{k}(0)=0$ for $k>1$. This suggests the use of a first order operator together with the boundary form $B_{1} y=y(1)-y(0)$. Now selfadjointness in a first order operator requires the presence of the imaginary unit $i$. Thus we choose $L y$ $=i y^{\prime}$ and $B_{1} y=y(1)-y(0)$. In this case the eigenvalues and eigenfunctions are $2 k \pi$ 
and $\exp [2 k \pi i x]$ for $k=0, \pm 1, \pm 2, \ldots$ In particular, 0 is a simple eigenvalue, and we may take $y_{0}=1$. Since $n=1$, we have $U_{1}=B_{1}$. Clearly,

$$
U_{1} L^{k} f=i^{k}\left[f^{(k)}(1)-f^{(k)}(0)\right] ;
$$

and a direct calculation shows that $i^{k} q_{k+1}=B_{k+1} /(k+1)$ ! for $k=0,1,2, \ldots$ Then

$$
\left(U_{1} L^{k} f\right) q_{k+1}(x)=\left[f^{(k)}(1)-f^{(k)}(0)\right] \frac{B_{k+1}(x)}{(k+1) !}, \quad k=0,1,2, \ldots
$$

Since $\left(f, y_{0}\right) y_{0}(x)=\int_{0}^{1} f(t) d t$, we see that the function $h_{p}$ in Theorem 2 is

$$
h_{p}(x)=\sum_{k=0}^{p-1}\left[f^{(k)}(1)-f^{(k)}(0)\right] \frac{B_{k+1}(x)}{(k+1) !}+\int_{0}^{1} f(t) d t .
$$

Lastly, the function $g_{p}(x)$ is given by

$$
g_{p}(x)=\sum_{k=-\infty ; k \neq 0}^{\infty} \frac{1}{(2 k \pi)^{p}}\left\{\int_{0}^{1} i^{p} f^{(p)}(t) e^{2 k \pi i t} d t\right\} e^{2 k \pi i x}
$$

By regrouping terms, one can show that this doubly infinite series reduces to the Fourier series in (1.3). We conclude that Theorem 2 reduces to the Lanczos representation.

(3) The three approximation methods studied by Jones and Hardy [4] are also special cases of our results. The reader may verify that Method I arises from Theorem 2 by considering the interval $-1 \leqslant x \leqslant 1$ and choosing $L y=i y^{\prime}$ and $B_{1} y=U_{1} y=$ $y(1)-y(-1)$. Method II is the Lidstone representation (1.4), and Method III is obtained from Theorem 2 by taking $L y=-y^{\prime \prime}, B_{1} y=y^{\prime}(0), U_{1} y=y^{\prime}(0)-y^{\prime}(1)$, and $B_{2} y=U_{2} y=y^{\prime}(1)$.

(4) A representation closely related to the Lanczos representation has been noted by Lyness [5]. Here, one employs Euler, rather than Bernoulli, polynomials. This method results from Theorem 2 by taking $L y=i y^{\prime}$ and $B_{1} y=U_{1} y=y(0)+y(1)$.

(5) Let $E(x)$ denote the error function with normalized variable,

$$
E(x)=\operatorname{erf}(2 x)=\frac{2}{\sqrt{\pi}} \int_{0}^{2 x} e^{-t^{2}} d t, \quad 0 \leqslant x \leqslant 1 .
$$

Jones and Hardy [4] have given extensive tabulations of the error arising from approximating $E(x)$ with their Methods II and III (see example (3) above). These methods yield an approximate representation of $\operatorname{erf}(2 x)$ in terms of finitely many polynomials and trigonometric functions. In the present example, we introduce an expansion which gives the same sort of result. Our calculation of Fourier coefficients is based on the trapezoidal rule, using 200 nodal points. The amount of calculation required is thus essentially the same as in [4], except that our use of the reduction formula (2.10) and integration by parts avoids the evaluation of $\operatorname{erf}(2 x)-h_{p}(x)$ in the trapezoidal rule.

Since $E(x)$ is an odd function we have $E^{(2 k)}(0)=0, k=0,1,2, \ldots$ This suggests the choice $L y=-y^{\prime \prime}$ and $B_{1} y=y(0)$, for then we have the simplification $B_{1} L^{k} E=0$ for all $k$. At the right endpoint $x=1$, we select $B_{2} y=y^{\prime}(1)$, so that 
$B_{2} L^{k} E=(-1)^{k} E^{(2 k+1)}(1), k=0,1,2, \ldots$ Thus we avoid calculating erf(2). Now the boundary-value problem

$$
-y^{\prime \prime}=\lambda y, \quad y(0)=y^{\prime}(1)=0
$$

has eigenvalues and eigenfunctions $\lambda_{k}=((2 k-1) \pi / 2)^{2}$ and $y_{k}(x)=\sqrt{2} \sin \sqrt{\lambda_{k}} x$, $k=1,2,3, \ldots$ Thus Theorem 1 applies in this case, and the "approximate representation" (3.1) reduces to

$$
E(x) \cong \sum_{k=0}^{p-1}(-1)^{k} E^{(2 k+1)}(1) p_{2 k+2}(x)+\sum_{k=1}^{m} \frac{(-1)^{p}\left(E^{(2 p)}, y_{k}\right)}{\lambda_{k}^{p}} y_{k}(x),
$$

The first few polynomials $p_{k}(x)$ are

$$
\begin{array}{ll}
p_{1}(x)=1, & p_{2}(x)=x, \\
p_{3}(x)=-\frac{x^{2}}{2}+x, & p_{4}(x)=-\frac{x^{3}}{6}+\frac{x}{2}, \\
p_{5}(x)=\frac{x^{4}}{24}-\frac{x^{3}}{6}+\frac{x}{3}, & p_{6}(x)=\frac{x^{5}}{120}-\frac{x^{3}}{12}+\frac{5 x}{24}, \text { etc. }
\end{array}
$$

A detailed error analysis of (3.2) is not wholly germane to this paper. Let it suffice, rather, to point out that there exist constants $M_{p}, p=1,2,3, \ldots$, such that

$$
\left|(-1)^{p}\left(E^{(2 p)}, y_{k}\right) y_{k}(x)\right| \leqslant M_{p},
$$

for $k=1,2,3, \ldots, p=1,2,3, \ldots$, and $0 \leqslant x \leqslant 1$. A simple application of the integral test to the terms omitted in (3.2) then shows that the error in (3.2) is less than

$$
\frac{M_{p}}{2(2 p-1)}\left(\frac{2}{\pi}\right)^{2 p}\left(\frac{1}{2 m-1}\right)^{2 p-1} .
$$

The table below compares, at evenly spaced points in $[0,2]$, the values listed in [1] for erf $(x)$, those calculated from the Fourier sine series (2.2), and those calculated from (3.2) with $p=3$ and $m=9$. The column headed $\operatorname{erf}(x)$ lists the values found in [1], the column headed $(0,9)$ contains the results of $(2.2)$ truncated after 9 terms, and the column headed $(3,9)$ gives the results of $(3.2)$ with $p=3$ and $m=9$. The error columns give the deviations of $(0,9)$ and $(3,9)$ from the erf $(x)$ column.

The Fourier coefficients were determined from (2.10), which reduces, in this case, to

$$
\lambda_{k}^{-p}\left(L^{p} E, y_{k}\right)=\left(E, y_{k}\right)-\left(p_{2}, y_{k}\right) \sum_{j=0}^{p-1} \frac{(-1)^{j} E^{(2 j+1)}(1)}{\lambda_{k}^{j}} .
$$

The values of $E^{\prime}(1), E^{\prime \prime \prime}(1)$ and $E^{(v)}(1)$ are found by direct computation. Similarly,

$$
\left(p_{2}, y_{k}\right)=\sqrt{2} \int_{0}^{1} x \sin \left(\sqrt{\lambda_{k}} x\right) d x=\frac{\sqrt{2}(-1)^{k+1}}{((2 k-1) \pi)^{2}}
$$

Finally, a simple integration by parts gives

$$
\left(E, y_{k}\right)=\frac{8}{(2 k-1)} \sqrt{2} / \pi \int_{0}^{1} e^{-4 x^{2}} \cos \left(\sqrt{\lambda}_{k} x\right) d x
$$

These values, used in both (2.2) and (3.2), were obtained by double-precision machine computation, using the trapezoid rule with 200 nodal points. 


\begin{tabular}{|r|c||c|c||c|c|}
\hline \multicolumn{1}{|c||}{$x$} & $\operatorname{erf}(x)$ & $(0,9)$ & error & $(3,9)$ & error \\
\hline \hline .2 & .22270259 & .22266823 & $343.6 \times 10^{-7}$ & .22270264 & $.5 \times 10^{-7}$ \\
.4 & .42839236 & .42845094 & $585.8 \times 10^{-7}$ & .42839245 & $.9 \times 10^{-7}$ \\
.6 & .60385609 & .60379204 & $640.5 \times 10^{-7}$ & .60385620 & $1.1 \times 10^{-7}$ \\
.8 & .74210096 & .74214850 & $475.4 \times 10^{-7}$ & .74210109 & $1.3 \times 10^{-7}$ \\
1.0 & .84270079 & .84269180 & $89.9 \times 10^{-7}$ & .84270089 & $1.0 \times 10^{-7}$ \\
1.2 & .91031398 & .91026847 & $455.1 \times 10^{-7}$ & .91031406 & $.8 \times 10^{-7}$ \\
1.4 & .95228512 & .95239414 & $1090.2 \times 10^{-7}$ & .95228517 & $.5 \times 10^{-7}$ \\
1.6 & .97634838 & .97617196 & $1764.2 \times 10^{-7}$ & .97634841 & $.3 \times 10^{-7}$ \\
1.8 & .98909050 & .98936261 & $2721.1 \times 10^{-7}$ & .98909053 & $.3 \times 10^{-7}$ \\
2.0 & .99532227 & .99430658 & $10156.9 \times 10^{-7}$ & .99532216 & $1.1 \times 10^{-7}$ \\
\hline
\end{tabular}

Department of Mathematics

Virginia Polytechnic Institute and State University

Blacksburg, Virginia 24061

1. M. ABRAMOWITZ \& I. A. STEGUN (Editors), Handbook of Mathematical Functions With Formulas, Graphs and Mathematical Tables, Nat. Bur. Standards Appl. Math. Ser., no. 55, Superintendent of Documents, U. S. Government Printing Office, Washington, D. C., 1964. MR 29 \#4914.

2. R. P. BOAS \& R. C. BUCK, Polynomial Expansions of Analytic Functions, Ergebnisse der Mathematik und ihrer Grenzgebiete, N. F., Band 19, Academic Press, New York; Springer-Verlag, Berlin, 1964. MR 29 \#218.

3. E. A. CODDINGTON \& N. LEVINSON, Theory of Ordinary Differential Equations, McGraw-Hill, New York, 1955. MR 16, 1022.

4. W. B. JONES \& G. HARDY, “Accelerating convergence of trigonometric approximations," Math. Comp., v. 24, 1970, pp. 547-560. MR 43 \#2823.

5. C. LANCZOS, Discourse on Fourier Series, Hafner, New York, 1966. MR 33 \#772.

6. J. N. LYNESS, "Computational techniques based on the Lanczos representations,"

Math. Comp., v. 28, 1974, pp. 81-123. MR 48 \#12777.

7. J. K. SHAW, "Series expansions and linear differential operators," SIAM J. Math. Anal., v. 7, 1976, pp. 311-331. 